\title{
El uso de tecnologías como factor del desarrollo socioafectivo en niños y jóvenes estudiantes en el noroeste de México
}

The use of technology as a factor of the affective development in children and young students in the Northwest of Mexico

O uso de tecnologias como fator de desenvolvimento social e emocional de crianças e jovens estudantes no noroeste do México

DOI: http://dx.doi.org/10.23913/ricsh.v6i11.113

Leonor Antonia Espinoza Núñez

Universidad Autónoma de Sinaloa, México leonor_espinoza63@hotmail.com

René Rodríguez Zamora

Universidad Autónoma de Sinaloa, México

rene.rodriguez@info.uas.edu.mx

\section{Resumen}

En el presente artículo se analiza la relación que existe entre el acercamiento al uso de las tecnologías y el desarrollo socioafectivo en los niños y jóvenes de entre 6 y 18 años de edad, se identifica en qué medida se tiene acercamiento a las variantes de estas tecnologías, así como las principales características del desarrollo social y emocional que manifiestan dichos niños y jóvenes. Se utilizó un enfoque de tipo ex post-facto con un nivel de alcance descriptivo de las unidades de análisis: alfabetización digital, socialización, y desarrollo emocional, tomando como muestra sujetos del nivel básico, medio básico y medio superior de la ciudad de Mazatlán en el estado de Sinaloa, México.

Se encontró que los niños menores de 12 años siguen prefiriendo la convivencia de manera presencial ya que expresaron estar más habituados a desarrollar lazos de amistad de manera 
personal, y en este sentido asumen el uso de los medios virtuales fundamentalmente con propósitos lúdicos y como un complemento que no sustituye el contacto directo con sus grupos de amigos. Con respecto a los jóvenes, se mostraron con gran capacidad de establecer relaciones con otras personas, particularmente con quienes son de género opuesto en la búsqueda de lograr lazos afectivos de pareja y/o de identidad sexual. Finalmente, con respecto al desarrollo emocional, los niños manifestaron seguridad y satisfacción como dos aspectos que se generan en ellos a partir del uso de la navegación por internet y de las redes sociales, destacando que no se observó una dependencia dado que no muestran frustración ante la falta del uso de estos medios tecnológicos. Por el contrario, la información con la que interactúan les sirve como un estímulo emocional positivo que evita el enojo y la agresividad. En el caso de los jóvenes, se mostraron muy satisfechos de hacer uso de las redes sociales como medio para expresar sus emociones de manera activa y manifestaron poca capacidad de reacción violenta o agresiva ante lo que observan en dichas redes.

Palabras clave: Comunicaciones, Desarrollo Emocional, Influencia de la Tecnología, Socialización, Tecnologías de la Información.

\section{Abstract}

This article analyzes the relationship between the approach to the use of technologies and the development of affective in children and young people between 6 and 18 years old, identifies to what extent is closer to the variants of these technologies, as well as the main features of the social and emotional development that these children and young people. It used an approach type ex post-facto with a level of descriptive scope of the analysis units: digital literacy, socialization, and emotional development, taking as subjects of the Basic, between basic and upper level of the city of Mazatlan in Sinaloa State, Mexico.

We found that children under 12 years old are still preferring coexistence in person since they expressed being more used to develop bonds of friendship in a personal way, and thus assume the use of virtual media primarily for recreational purposes and as an add-on that does not replace direct contact with their groups of friends. With regard to young people, were with large capacity of establishing relationships with others, particularly with those 
who are gender opposite in the pursuit of achieving emotional ties partner or sexual identity. Finally, with regard to emotional development, children expressed safety and satisfaction as two aspects that are generated in them from the use of the navigation by internet and social networks, stressing that there was a unit since they do not show frustration at the lack of the use of these technologies. On the contrary, the information with which they interact serves as a positive emotional stimulation that avoids anger and aggression. In the case of young people, were very pleased to make use of social networks as a means to express their emotions in an active way and demonstrated limited capacity for violent or aggressive reaction to what observed in these networks.

Key words: communications, emotional development, influence of technology, socialization, Information technologies.

\section{Resumo}

Neste artigo, a relação entre a abordagem do uso da tecnologia e desenvolvimento social e emocional de crianças e jovens entre os 6 e os 18 anos, identifica a medida em que analisa você tem abordagem para variantes destas tecnologias bem como as principais características do desenvolvimento social e emocional que se manifestam estas crianças e jovens. uma abordagem ex post facto, com um nível de alcance descritivo das unidades de análise foi utilizado: a literacia digital, socialização e desenvolvimento emocional, tomando como amostra sujeita nível básico, médio básica e média alta da cidade de Mazatlan no estado de Sinaloa, no México.

Verificou-se que as crianças menores de 12 anos ainda preferem viver juntos em pessoa desde que expressaram sua mais acostumados a desenvolver laços de amizade, pessoalmente, e, nesse sentido, assumem o uso de meios virtuais, principalmente para fins recreativos e como complemento ele não substitui o contato direto com grupos de amigos. No que diz respeito aos jovens, eles foram mostrados com grande capacidade de estabelecer relacionamentos com outras pessoas, particularmente aqueles que são do sexo oposto na busca para alcançar parceiro de ligação e / ou identidade sexual. Finalmente, no que diz respeito ao desenvolvimento emocional, as crianças demonstraram segurança e satisfação 
como dois aspectos que são gerados neles a partir do uso de navegação na Internet e as redes sociais, notando que a dependência não foi observado uma vez que nem mostrar frustração a falta de uso desses meios tecnológicos. Pelo contrário, a informação que interagem serve como estímulo emocional positivo que evita raiva e agressão. No caso dos jovens, eles foram muito prazer em fazer uso de redes sociais como um meio de expressar suas emoções e demonstrar ativamente pouca capacidade para violento ou agressivo para o que observam nestes reacção redes.

Palavras-chave: comunicações, desenvolvimento emocional, influência da tecnologia, a socialização, a tecnologia da informação.

Fecha Recepción: Junio 2016 Fecha Aceptación: Diciembre 2016

\section{Introducción}

Los jóvenes de las nuevas generaciones crecieron con una tecnología que las ha definido por su familiaridad y confianza en las TIC. Echenique (2012) se refiere a ellos como los jóvenes que han crecido en un mundo digital y esperan utilizar estas herramientas para sus entornos avanzados de aprendizaje, como parte de su vida cotidiana, sus pasatiempos y su forma de interacción; se rodean del uso de videjuegos, reproductores de música, cámaras, mensajería instantánea y multimedia, lo que les ha dado la posibilidad de desarrollar habilidades en torno al procesamiento de información.

Prensky (2001) denomina nativos digitales a los estudiantes de hoy, ellos representan las primeras generaciones que han crecido con esta nueva tecnología. Han pasado toda su vida rodeados de, y usando ordenadores, lo que los hace pensar y procesar la información de manera diferente a los inmigrantes digitales, refiriendo a todos aquellos que sin nacer en el mundo digital, en algún momento han adoptado muchos o la mayoría de los aspectos de la nueva tecnología. White (2008), por otro lado, recomienda no categorizar a los usuarios como nativos o inmigrantes digitales, a partir de las tecnologías utilizadas sino a partir de la forma de su utilización. Para ello introduce el concepto de residente como la persona que 
vive un porcentaje de su vida en línea, y visitante como el que utiliza la web de manera organizada como una herramienta cuando es necesario.

La aparición de nuevas tecnologías ha producido no sólo una revolución económica sino también de carácter psicológica y social, pues nos ofrece con el acceso a la información proveniente de cualquier lugar, nuevas formas de convivencia y de relaciones interpersonales donde se traspasan las fronteras de la distancia, de las edades, de las clases sociales y de los géneros, entre otros.

Las nuevas tecnologías al alcance de los niños y jóvenes desde su nacimiento, ofrecen no solo nuevas posibilidades de acceso a la información y comunicación, sino también nuevos paradigmas de relación con el contexto social, a través de la modificación y generación de nuevas habilidades socioafectivas. Es importante conocer de qué manera las formas de socialización se han transformado a partir del uso de la tecnología. Por ello, en este estudio se da a conocer el nivel y el tipo de uso de las tecnologías entre los niños y jóvenes, así como el impacto en su forma de vida.

Además se identifica la relación que existe entre el acercamiento al uso de las TIC y el desarrollo socioafectivo en niños y jóvenes estudiantes de nivel educativo básico, medio básico y medio superior. Esto abarca la precisión del acercamiento al uso de las tecnologías, la identificación de las características del desarrollo social y emocional mediante el uso de las TIC. Se encontró que los niños no sustituyen las formas de socialización al contacto físico sino que amplían la gama de interacción siendo el juego y el entretenimiento el principal objetivo. Mientras que en el caso de los jóvenes, es un medio para desarrollar habilidades sociales y emocionales, pues la interactividad virtual aparece como un potenciador de las capacidades de socialización.

\section{Método}

El enfoque metodológico utilizado es cuantitativo de tipo ex post-facto (Bizquerra, 2004) con un nivel de alcance descriptivo de las unidades de análisis: alfabetización digital, socialización, y desarrollo emocional. El universo de la investigación está constituido por nativos digitales e inmigrantes digitales de ambos sexos comprendidos entre los 6 y los 18 años de edad, estudiando desde el nivel básico hasta el nivel superior en el Estado de 
Sinaloa, México. La población con la que se trabajó para diseñar la muestra fué la de individuos de nivel económico medio con acceso a servicios de conexión a internet en la zona urbana de Mazatlán, Sinaloa, como fuente primaria para la recolección de datos se utilizó un único cuestionario el cual fue validado por expertos en el área de Tecnología y Psicología, además de que se aplicó el coeficiente Alfa de Cronbach. Como fuentes secundarias para el diseño de la muestra se utilizó el anuario estadístico y geográfico de Sinaloa (INEGI, 2014) y el diagnóstico del contexto sociodemográfico elaborado por el Centro de Integración Juvenil de Mazatlán (CIJ, 2008). A partir de los datos obtenidos de estas fuentes secundarias se calculó el tamaño de una muestra aleatoria representativa de 150 sujetos, utilizando la siguiente ecuación:

$$
n=\frac{N Z^{2} S^{2}}{d^{2}(N-1)+Z^{2} S^{2}}
$$

El cuestionario diseñado estuvo compuesto de 42 puntos, que abordaron los principales atributos de las unidades de análisis.

\section{Resultados}

\section{A) Alfabetización digital de niños y jóvenes}

Las generaciones jóvenes son las más susceptibles a generar las nuevas formas de comportamiento social y cultural de la sociedad contemporánea basadas en el uso habitual de las distintas tecnologías digitales, así recurrimos en este estudio al término de Alfabetización Digital para referirnos a la forma en que el niño y el joven interactúa y aprende sus códigos de comunicación básicos mediante su relación con la tecnología. Para cualquier joven la telefonía móvil y la navegación por Internet son experiencias cotidianas, así también para los niños el uso de algún juguete digital programable, pasa a ser la forma de juego de interacción social más común, por ello su nivel de manejo y operatividad sobre los recursos electrónicos alcanza niveles extraordinarios ya que es su forma de entender y expresarse con todo lo que le rodea, sin embargo es muy determinante el acceso de la población a los recursos digitales, y sobre todo el nivel de manejo que tiene con el mismo. 
De acuerdo al estudio realizado por la AMIPCI (2015) sobre el internauta mexicano, del total de encuestados el $12 \%$ de la población oscila entre los 6 a 12 años de edad, y el 26\% de los 13 a los 18 años, haciendo un total de $38 \%$ de usuarios de medios digitales entre los más jóvenes. Los datos también muestran que el lugar de acceso más usual sigue siendo el hogar, lo que indica que niños y jóvenes mantienen entre sus hábitos preferentes de permanencia en el hogar la interacción con algún elemento digital para estar conectado de manera virtual con los demás. Es decir, cada vez se están utilizando los dispositivos digitales a más temprana edad.

En el estudio realizado se presenta la agrupación de variables que explican cómo se sienten los niños de 6 a 12 años de edad con respecto al dominio, familiaridad y uso de los medios digitales, esta agrupación de variables no reporta valores altos, lo que implica un dominio no alto del uso de los implementos tecnológicos, encontrando que todos los valores se encuentran en el rango de la media, $(\mathrm{Xx}=7.83)$ es decir los niños se sienten regularmente capaces de saber utilizar los implementos digitales como la computadora y el celular, siendo los más usuales las consolas de videojuegos y las tabletas. Con respecto a la red de Internet también expresan saber usarla de manera regularmente aceptable, utilizándola más con fines de diversión y entretenimiento que con fines de establecer comunicación con sus grupos de convivencia, como amigos y familia. Declara dominar medianamente redes sociales como Facebook, y WhatsApp, reconociendo que tiene escaso conocimiento $(X=4.16)$ en el uso de medios de interlocución o comunicación como Skype y Twitter.

Figura 1. El uso de los medios digitales en los niños.

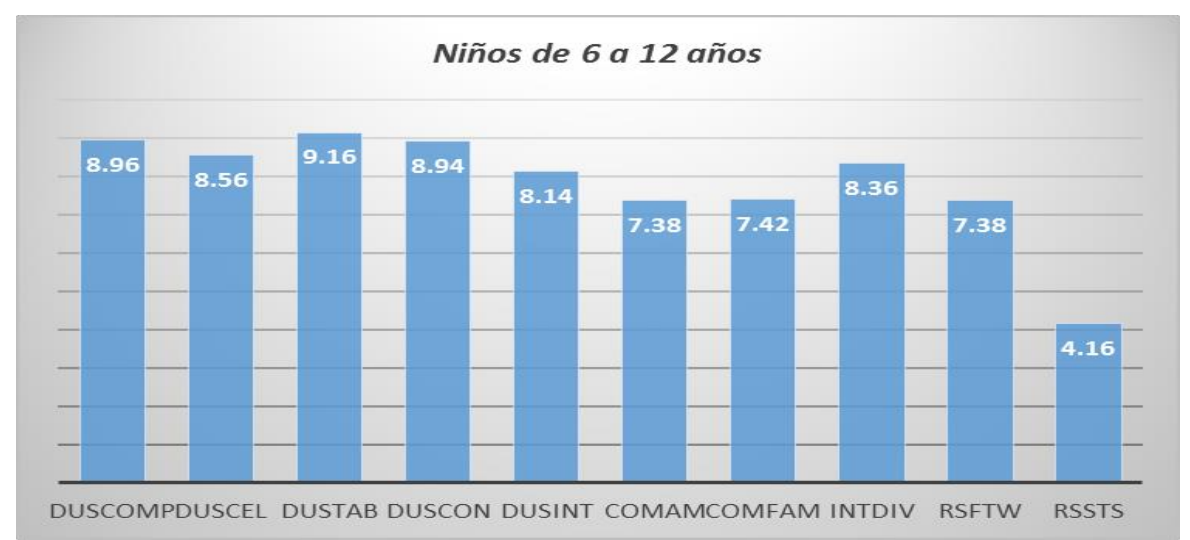

Fuente: Elaboración propia. 
Como se observa en la Figura 1, los niños no se sienten muy seguros e interesados en aprender el manejo de la computadora para las llamadas presenciales o para estar en comunicación con sus grupos. Su interés está centrado en el manejo tabletas y dispositivos que contengan juegos interactivos, por lo que se infiere que los niños de 6 a 12 años se inclinan más por el uso de dispositivos como un medio lúdico que los mantiene en contacto con los suyos como medio de socialización.

Con respecto a los adolescentes de 13 a 18 años de edad (ver Figura 2), considerando el comportamiento de esta agrupación de variables $(X x=7.78)$ tampoco se registran valores altos, por lo que los jóvenes muestran de manera regularmente aceptable el dominio de los diferentes dispositivos digitales, reportan que se sienten más capaces $(X=9.12)$ en la utilización de las funciones que ofrece el celular como dispositivo móvil. Los resultados muestran que estar en conectividad con Internet, estar en comunicación con los amigos mediante las redes sociales más usuales como Facebook y WhatsApp son sus principales intereses.

Figura 2. El uso de los medios digitales en los adolescentes.

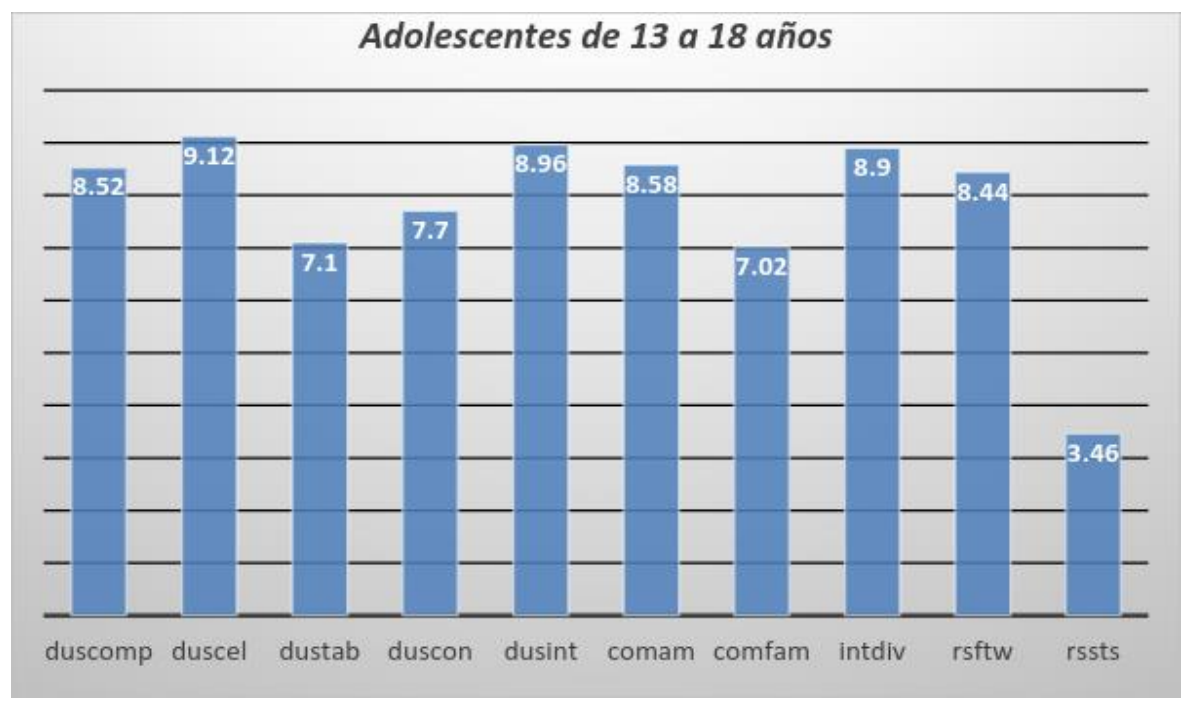

Fuente: Elaboración propia.

La gráfica nos muestra que el uso del celular y la conectividad a Internet son de las principales necesidades en la adolescencia, situación que ha impactado en la forma de socialización que los jóvenes adolescentes han establecido, lo que para los padres puede 
representar un dispositivo de información y comunicación para estar actualizado en el conocimiento, para los jóvenes se ha convertido en un estilo diferente para el desarrollo psicosocial. Para Coll y Monereo (2008) la alfabetización digital es el proceso mediante el cual las personas adquirimos los conocimientos y las competencias necesarios para convertirnos en miembros de un grupo que comparte una cultura determinada, es por ello que en la adolescencia las tecnologías se han convertido en una poderosa herramienta que potencía el desarrollo de nuevas habilidades, construyendo entre sus usos y hábitos generados a partir de las condiciones proporcionadas por el mundo adulto, una nueva cultura de interacción digital.

Se infiere así, que cada vez es más habitual que niños y adolescentes dispongan de computadoras en los hogares, posteriormente de dispositivos portátiles, tales como laptops tabletas, reproductores de audio, videoconsolas portátiles $\mathrm{y}$, por supuesto, como se muestra en los resultados, teléfonos móviles, lo que permite que la conectividad se prolonga a lugares fuera del hogar.

Sendín, Gaona y García (2014) en su estudio realizado en España constatan la cada vez menor edad de acceso a Internet, que se sitúa en los ocho años, junto a las 3 ó 4 horas diarias de conexión que comentan. Comparado esto con los resultados del presente estudio se observa que aunque las tendencias en la alfabetización digital, tienen un factor cultural, los grupos de edad son determinantes.

\section{B) El proceso de socialización y las tecnologías}

Desde que el ser humano se pone en contacto con el mundo, establece un tipo de interacción con él, desde que nace desarrolla un primer vínculo con la madre, que posteriormente extiende a la familia, los amigos y todo su contexto. Con ese medio interactúa y aprende de esas acciones, inicialmente es la imitación la base a partir de la cual construye sus principales patrones de conducta, y crea necesidades sociales mediante la convivencia y el manejo del contacto con los otros, a partir de sus grupos genera como principal necesidad un sentido de pertenencia indispensable para la formación de su personalidad. De esta manera las transformaciones del contexto van moldeando las pautas para el establecimiento de nuevos patrones de conducta y de relaciones sociales. Bernete (2012) en su estudio habla de 
nuevas formas de crecer y de generar vínculos, sin que ello implique la destrucción o el abandono de las anteriores, sino que con las innovaciones tecnológicas se van implementando nuevas formas de obtener información y recreación entre los niños y adolescentes.

En este sentido, es importante conocer los principales hábitos de los niños que les permiten el desarrollo de habilidades y competencias sociales. Así como la relación entre la etapa de desarrollo de acuerdo a la edad y la aparición del interés por usar por primera vez Internet como medio de acceso a la información y socialización.

Figura 3. Edad de inicio en el uso de herramientas tecnológicas.

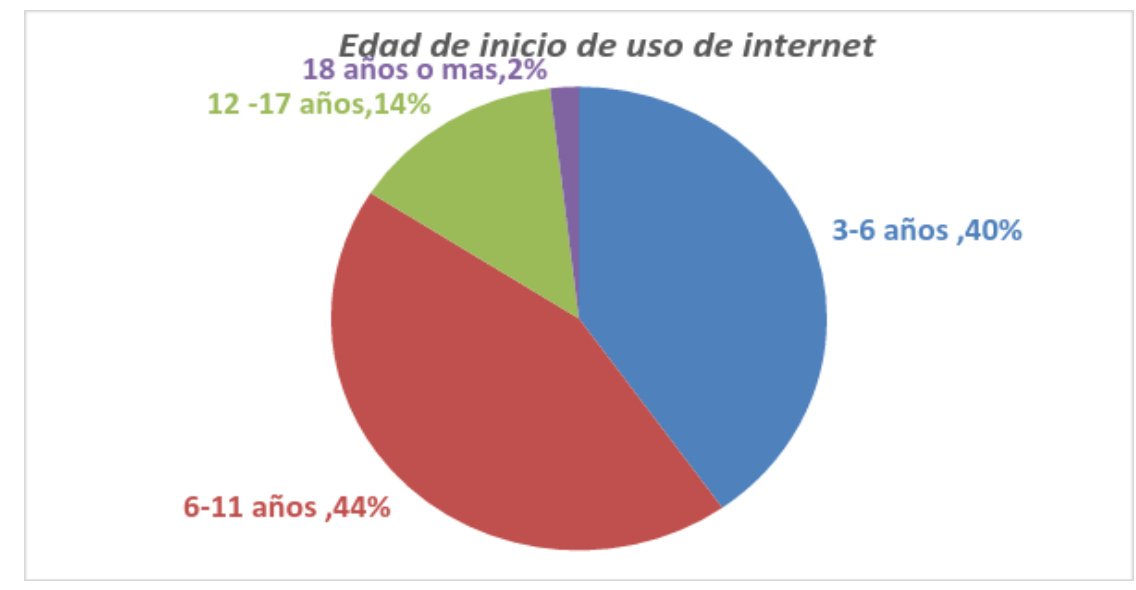

Fuente: $11^{\circ}$ estudio sobre los hábitos de los usuarios de Internet en México 2015.

Como se observa en la gráfica de la Figura 3, los niños de 3 a 6 años representan el 40\%, mientras que los niños de 6 a 11 años el 44\%, lo que muestra que la edad de inicio oscila entre los niños de 3 a 11 años, a partir de los 12 años el porcentaje va decreciendo, situación que habla del perfil de las nuevas generaciones que están a muy temprana edad familiarizados con los medios virtuales como formas de acceso a la información.

Se describe en este estudio con más detalle el comportamiento de la agrupación de variables que tienen que ver con las preferencias de socialización de los niños inicialmente de 6 a 12 años utilizando las tecnologías. Es decir, explica los hábitos de uso de redes sociales como medio de interacción y/o entretenimiento para los niños en este período del desarrollo. Se 
describen en este apartado las características del uso de redes sociales, la interlocución y el juego, así como sus preferencias en la convivencia social.

Figura 4. Hábitos de socialización en los niños de 6 a 12 años.

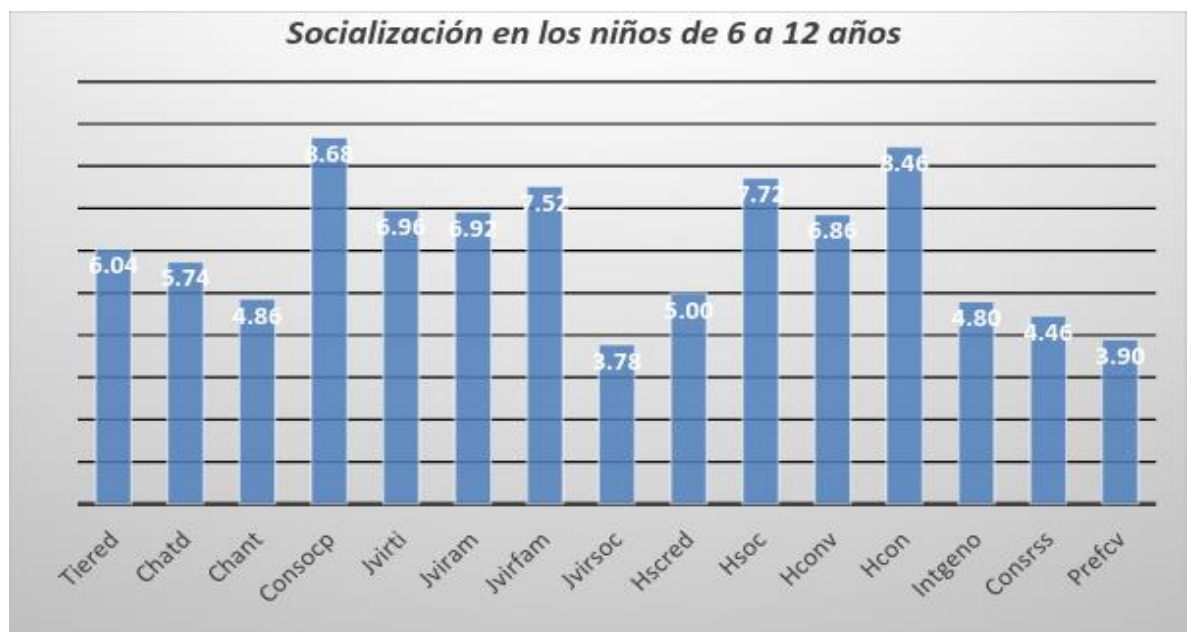

Fuente: Elaboración propia.

Los niños muestran (ver Figura 4) de acuerdo a los valores de la media $(X x=8.68)$, que prefieren la convivencia con los suyos de manera presencial, es decir, necesitan más del contacto con las personas que les rodean para sentirse seguros. Ya que expresa estar más acostumbrado (Ls $\geq 7.66$ ) a hacer amistades de manera personal, manifiestan también sentirse plenamente más hábiles para entablar conversaciones con los otros de manera presencial. Declaran que acceden de manera regular a las redes sociales para contactar, utilizan de manera regular las redes y los juegos virtuales para estar en contacto y convivir preferentemente con la familia.

Demuestran sin embargo que cuando usan las redes permanecen muy poco tiempo $(\mathrm{Li} \leq 4.56)$ en contacto, escasamente dejan de hacer otras actividades por estar en el Chat, ya que muy pocas veces dejan la oportunidad de disfrutar el tiempo de convivencia personal por atender los medios virtuales.

Es decir, los niños a muy temprana edad tienen acceso a las herramientas tecnológicas, y por lo tanto se familiarizan rápidamente con el manejo de estos medios para estar en contacto con las personas cercanas interactuando mediante el juego, pero siguen aún más habituados 
al contacto personal como medio de socialización, lo que implica que los medios virtuales no sustituyen el contacto directo con sus grupos pero si complementan y representan una forma de abrir su campo de acción social.

Con respecto a los jóvenes de 13 a 18 años, $(X x=6.13)$ a diferencia de los niños menores, declaran tener mucha habilidad ( $\mathrm{Ls} \geq 7.78$ ) para platicar en medios virtuales, ya que se sienten con una gran capacidad de establecer relaciones en diálogo con otras personas, así mismo, una de sus preferencias más apremiantes es relacionarse con el género opuesto estableciendo una relación de aceptación, con él o ella. De tal forma que pueden llegar a construir relaciones de noviazgos a través de las herramientas virtuales.

Sin embargo, muestran escaso interés $(\mathrm{Li} \leq 4.48)$ en los videojuegos como formas de socialización con sus iguales, ya que muy pocas veces recurren a los videojuegos para compartir con los amigos o para estar en contacto con alguien más en línea.

Figura 5. Hábitos de socialización en adolescentes de 11 a 18 años.

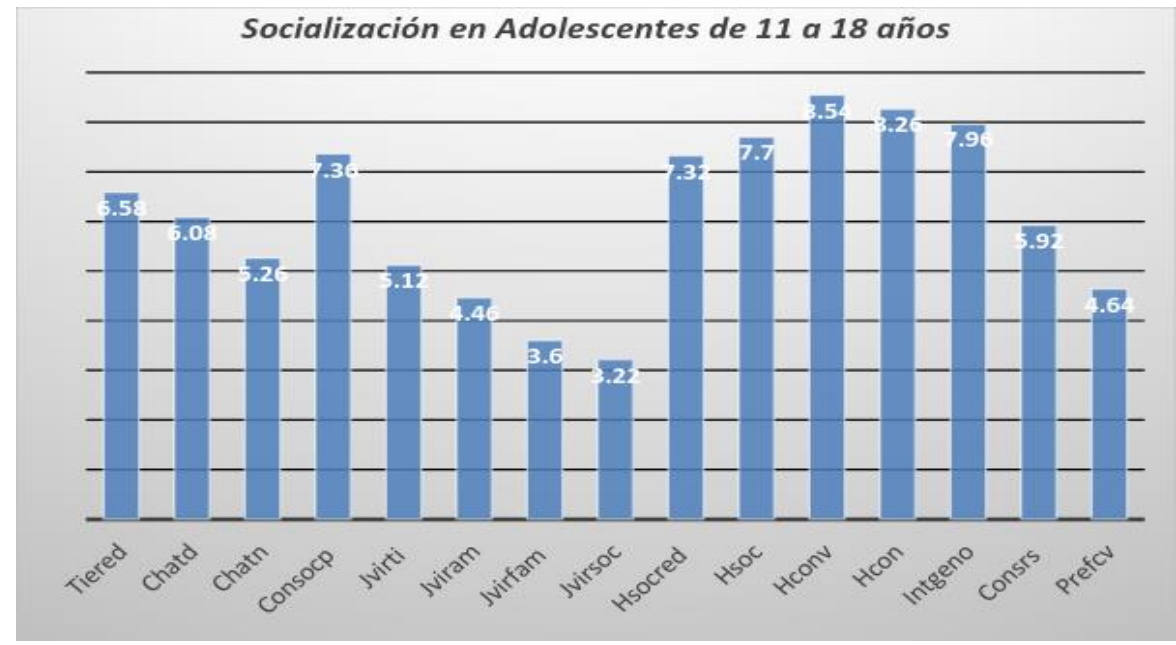

Fuente: Elaboración propia.

Se infiere que en el caso de los niños más pequeños las formas de socialización mediante el uso de las tecnologías no sustituyen al contacto físico o personal sino que representan la posibilidad de ampliar la gama de interacciones al tener acceso a la información y a las redes sociales, la interacción de los niños de 3 a 6 años de edad con las tecnologías están más orientadas al entretenimiento y el juego. En el caso de los adolescentes (ver Figura 5) el 
uso de las herramientas se amplía y representa la oportunidad de establecer relaciones e interacción con sus iguales, generando nuevas pautas de comportamiento y de patrones en la interacción. Martínez y Espinar (2012) afirman que la no posesión de los dispositivos móviles implica, según los adolescentes, la exclusión y marginación entre iguales. De acuerdo a los datos obtenidos en este estudio, representa además la seguridad de ser aceptado por el género opuesto que es una de las necesidades psicosociales más demandantes en este período de edad en el que se convierte en un elemento para el fortalecimiento de la identidad.

\section{C) El desarrollo emocional y el uso de las tecnologías}

Las emociones representan un factor importante para el desarrollo social de los niños y jóvenes, al mismo tiempo que el desarrollo emocional es determinado por la combinación de una serie de factores que van desde lo innato y biológico hasta lo sociocultural, en este sentido, nos enfocamos a la gran diversidad de estímulos que le son proporcionados al sujeto por la complejidad del contexto en el que se desenvuelve, una complejidad que se ha multiplicado por la influencia de la tecnología. Se analizan entonces el conjunto de variables que explican cómo se sienten niños y jóvenes con respecto a su desarrollo emocional frente a la manera en que se relacionan con la tecnología.

Figura 6. Respuesta emocional en los niños de 6 a 11 años.

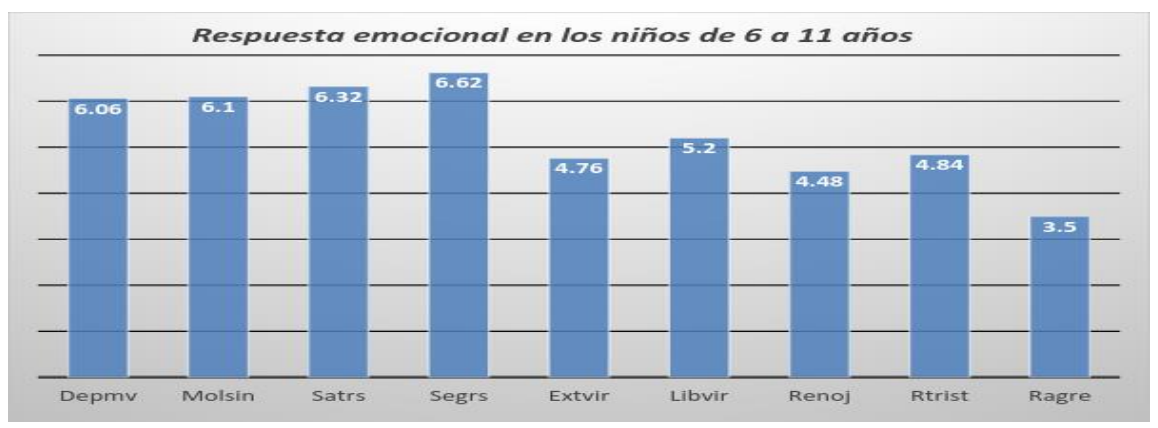

Fuente: Elaboración propia.

De acuerdo al comportamiento de la media para esta agrupación de variables $(X x=5.32)$, los niños reportan (ver Figura 6) que al estar en contacto con las tecnologías sienten mucha seguridad al navegar por Internet y en el uso de las redes sociales (Ls $\geq 6.34$ ), de hecho 
declaran sentirse muy satisfechos al estar interactuando en alguna de las redes sociales, con respecto a la dependencia generada por los medios virtuales manifiestan un regular control emocional ya que aunque admiten que les hace falta, no demuestran angustia ni desesperación si no están en contacto con ellos. Más aún, declaran escasas reacciones de enojo frente a cualquier situación provocada por la información de las tecnologías ( $\mathrm{Li} \leq 4.3$ ), de la misma manera manifiestan que en escasas ocasiones han reaccionado con agresividad o deseos de violencia, provocada por factores circunstanciales en las redes sociales y la información obtenida en los medios virtuales.

Los niños muestran agrado y preferencia al hábito de estar conectados en red o usar los medios digitales, lo que implica que son parte de sus intereses, sin embargo, no han generado dependencia a los mismos, pues pueden controlar sus emociones ante la falta de ellos sin generar frustración, es decir, reconocen que le son indispensables pero sin generarles descontrol emocional, por otra parte, la reacción psicológica y afectiva ante la información con la que interactúa le sirve de estímulo emocional sin llegar a ser un factor disparador significativo de emociones negativas como el enojo y la agresividad.

En el caso de los jóvenes adolescentes, se observa (ver Figura 7) que demuestran una gran satisfacción al estar en interacción con las redes sociales, a diferencia de los niños declaran haber desarrollado la competencia de la interacción virtual, lo que les permite expresarse emocionalmente con mucha facilidad para relacionarse con gente por medios virtuales, por otra parte, declaran que escasamente han reaccionado con respuestas agresivas o violentas hacia lo que sucede en las redes. 
Figura 7. Respuesta emocional de los adolescentes de 11 a 18 años de edad.

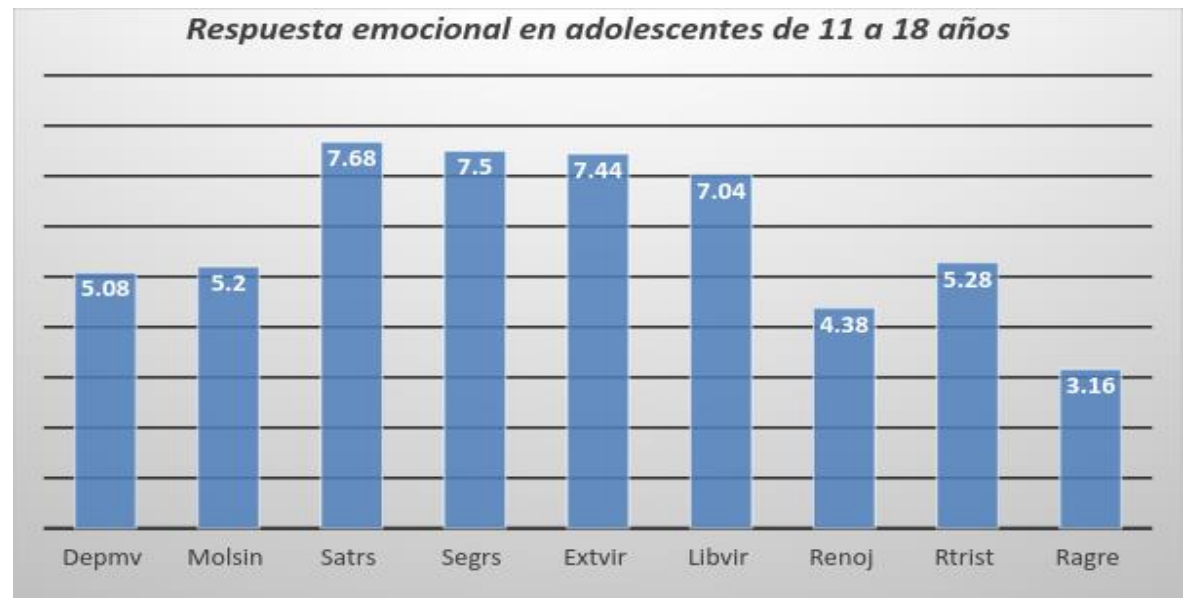

Fuente: Elaboración propia.

Los jóvenes adolescentes han desarrollado así mayores habilidades para socializarse con su grupo de iguales y expresar de esa manera sus emociones al entrar en contacto con los otros, siendo esta interacción virtual un factor importante para su desarrollo emocional. La oportunidad de la participación en la interactividad, principal característica de la Web 2.0, se convierte en factor potenciador de las emociones tanto en su capacidad de expresión como en su capacidad de producción y desarrollo, ya que el joven participa de modo activo, elaborando su propia producción. Martínez, Segura y Sánchez (2011) plantean la duda acerca de si el cerebro humano está preparado para producir emociones adecuadas a la velocidad con la que se expone a la información. De acuerdo a los datos se puede inferir que el contenido de las redes y los medios virtuales no afectan de manera significativa la promoción de emociones consideradas nocivas o destructivas en los niños y jóvenes.

\section{Discusión}

Existe en la actualidad un gran debate con respecto a la influencia de los medios digitales en el desarrollo de la psique en el período de la infancia y la adolescencia por ser la etapa fundamental de la construcción de la identidad, en torno a que han automatizado las reacciones de las personas. Ante tales reflexiones, Buckingham (2002) trata de establecer una reflexión equilibrada entre los temores sociales acerca de la influencia negativa y el exagerado optimismo de perfilar una generación electrónica. 
Si bien es cierto que el alcance de este estudio se circunscribe solamente a un segmento poblacional, los resultados obtenidos muestran, por un lado, que es necesario establecer políticas públicas que faciliten el acceso justo e igualitario a la información que el mundo produce, y el diseño de estrategias mediáticas para dirigir ese acceso hacia el progreso de la sociedad actual; y por otro lado, que las herramientas tecnológicas deben dejar de ser vistas bajo una mirada amenazadora y ser aprovechadas como elementos potenciadores no solo de un avance en las innovaciones informativas, sino también como un factor de desarrollo psicosocial del sujeto.

\section{Conclusiones}

En México, poco más de un tercio de los usuarios de medios digitales a través de Internet son niños y jóvenes de entre 6 y 18 años. A partir de este referente se obtuvieron diversos resultados con base en el análisis presentado en este documento. Entre estos resultados se encontró que los niños de entre 6 y 12 años utilizan mayormente las consolas de videojuegos y las tabletas porque sienten y expresan mayor habilidad para manejar estos dispositivos. Según expresaron, utilizan Internet principalmente para fines de entretenimiento, y medianamente para el uso de redes sociales.

No obstante lo antes mencionado, los niños se manifiestan muy interesados en aprender a utilizar dispositivos móviles como tabletas y celulares, o en su caso computadoras personales. Por otro lado, los jóvenes de entre 13 y 18 años se mostraron más seguros y capaces de utilizar el celular como dispositivo para conectarse y hacer uso de Internet para establecer redes de socialización con amigos. Existe una alfabetización digital medianamente aceptable, puesto que utilizan de manera parcial sólo algunos dispositivos y este uso fundamentalmente es para propósitos lúdicos en el caso de los niños, y de conexión para comunicarse con amigos en el caso de los jóvenes.

Así pues, el proceso de socialización a través del análisis mostró que los niños siguen prefiriendo la convivencia de manera presencial ya que expresaron estar más habituados a desarrollar lazos de amistad de manera personal, y en este sentido, asumen el uso de los medios virtuales como un complemento que no sustituye el contacto directo con sus grupos de amigos. Con respecto a los jóvenes, se sienten con gran capacidad de establecer 
relaciones con otras personas, particularmente con quienes son de género opuesto en la búsqueda de lograr lazos afectivos de pareja y/o de identidad sexual.

Con respecto al desarrollo emocional, los niños manifestaron seguridad y satisfacción como dos aspectos que se generan en ellos a partir de la navegación por Internet y uso de las redes sociales, destacando que no se observó una dependencia dado que no muestran frustración ante la falta del uso de estos medios tecnológicos. Por el contrario, la información con la que interactúan les sirve como un estímulo emocional positivo que evita el enojo y la agresividad. En el caso de los jóvenes, se mostraron muy satisfechos de hacer uso de las redes sociales como medio para expresar sus emociones de manera activa y manifestaron poca capacidad de reacción violenta o agresiva ante lo que observan en dichas redes. 


\section{Bibliografía}

Alarcia, Ó F., Bravo, I. D. (2013). Nativos digitales, inmigrantes digitales: Rompiendo mitos. Un estudio sobre el dominio de las TIC en profesorado y estudiantado de la Universidad de Lleida. Bordón. Revista De Pedagogía BRP, 65(2), 59-74. doi:10.13042/brp.2013.65204.

Alzina, R. B., Escoda, N. P. (2007). Las competencias emocionales. Educación XX1, 10(0). doi:10.5944/educxx1.1.10.297

Bernete, F. (2012). Usos de las TIC, Relaciones sociales y cambios en la socialización de las y los jóvenes. Revista Estudios de Juventud, juventud y nuevos medios de comunicación (88), 97-114.

Bizquerra, R. (2009). Metodología de la investigación educativa. Editorial La Muralla. España.

Buckingham, D. (2002). Crecer en la era de los medios electrónicos. Editorial Morata.

Buitrago, L. D., García, C. R., García, S. R. (2016, 03). Las TIC como Herramientas de Inclusión Social. 3C TIC 3C TIC Cuadernos De Desarrollo Aplicados a Las TIC, 5(1), 54-67. doi:10.17993/3ctic.2016.51.54-67.

Castells, M., Gimeno, C. M. (1997). La Era de la información: Economía, sociedad y cultura. Madrid: Alianza.

CIJ. (2008). Diagnóstico del contexto socio-demográfico del área de influencia del CIJ Mazatlán. Centro de Integración Juvenil A.C. Mazatlán, Sinaloa, México.

Coll, C., Monereo, C. (2008). Psicología de la educación virtual. Aprender y Enseñar con las Tecnologías de la Información y la Comunicación. Editorial Morata. España.

Domínguez, D. C. (2013). Los retos de la era de las TIC: Nativos digitales contra inmigrantes. Comunicación Y Medios, 0(23). doi:10.5354/0716-3991.2011.26336. 
Echenique, E. E. (2012). Hablemos de estudiantes digitales y no de nativos digitales. Revista de ciencias de la educación, 7-21.

Internet, A. M. (2015). $11^{\circ}$ Estudio sobre los hábitos de los usuarios de internet en México 2015. México.

Gutiérrez, J. C., Pisonero, C. G., Jiménez, A. G. (2014). Nuevos medios: Usos comunicativos de los adolescentes. Perspectivas desde los nativos digitales. Estudios Sobre El Mensaje Periodístico, 20(1). doi:10.5209/rev_esmp.2014.v20.n1.45231.

INEGI. (2014). Anuario Estadístico y Geográfico de Sinaloa 2014. Instituto Nacional de Estadística, Geografía e Informática. México.

Martinez, Á C. (2010). Pautas de crianza y desarrollo socioafectivo en la infancia. Divers.: Perspect. Psicol. Diversitas, 6(1), 111. doi:10.15332/s1794-9998.2010.0001.08.

Martínez, G. R., Espinar R. E. (2012). Adolescentes y tecnologías de la información y comunicación en España. Revista de Ciencias Sociales. 7(1), 109-122.

Martínez, R.E., Segura, G. R., Sánchez, M. L. (2011). El complejo mundo de la interactividad: emociones y redes sociales. Revista Mediterránea de Comunicación. 2(1).

Moreira, M. A., Martín, A. G., Fernández, F. V. (2012). Alfabetización digital y competencias informacionales. Barcelona: Ariel.

Núñez-Gómez, P., García-Guardia, M., Hermida-Ayala, L. (2012). Tendencias de las relaciones sociales e interpersonales de los nativos digitales y jóvenes en la web 2.0. doi:10.4185/rlcs-067-952-179-206

Pazos, A. B. (2003). Nuevos modos de construcción de la identidad en la sociedad internacional. Revista latina de comunicación social, 54.

Prensky, M. (2001). Digital Natives, Digital Immigrants Part 1. On the Horizon, 9(5), 1-6. doi:10.1108/10748120110424816. 
Rodrigo, R. S. (2011). Emociones y Nuevas Tecnologías en la Red. En M. F. Ortega, Nuevos Medios, Nueva Comunicación (págs. 858-868). Salamanca: Universidad de Salamanca.

Sendín, J., Gaona, C., García, A. (2014). Nuevos medios: usos comunicativos de los adolescentes. Perspectivas desde los nativos digitales. Estudios Sobre El Mensaje PeriodíStico, 20(1), 265-280. doi:10.5209/rev_ESMP.2014.v20.n1.45231.

Toledo, W. T. (2006). La construcción de la identidad y la apropiación de la tecnología en los medios. Questions , 1, 12.

White, D. S., Cornu, A. L. (2011, 08). Visitors and Residents: A new typology for online engagement. First Monday, 16(9). doi:10.5210/fm.v16i9.3171. 